\title{
Ion temperature anisotropy effects on threshold conditions of a shear-modified current driven electrostatic ion-acoustic instability in the topside auroral ionosphere
}

\author{
P. J. G. Perron ${ }^{1}$, J.-M. A. Noë ${ }^{1}$, K. Kabin ${ }^{1}$, and J.-P. St-Maurice ${ }^{2}$ \\ ${ }^{1}$ Department of Physics, Royal Military College of Canada, Kingston, Ontario, Canada \\ ${ }^{2}$ Institute of Space and Atmospheric Studies and Department of Physics and Engineering Physics, University of \\ Saskatchewan, Saskatoon, Saskatchewan, Canada \\ Correspondence to: P. J. G. Perron (patrick.perron@rmc.ca)
}

Received: 30 October 2012 - Revised: 29 January 2013 - Accepted: 18 February 2013 - Published: 7 March 2013

\begin{abstract}
Temperature anisotropies may be encountered in space plasmas when there is a preferred direction, for instance, a strong magnetic or electric field. In this paper, we study how ion temperature anisotropy can affect the threshold conditions of a shear-modified current driven electrostatic ion-acoustic (CDEIA) instability. In particular, this communication focuses on instabilities in the context of topside auroral F-region situations and in the limit where finite Larmor radius corrections are small. We derived a new fluidlike expression for the critical drift which depends explicitly on ion anisotropy. More importantly, for ion to electron temperature ratios typical of F-region, solutions of the kinetic dispersion relation show that ion temperature anisotropy may significantly lower the drift threshold required for instability. In some cases, a perpendicular to parallel ion temperature ratio of 2 and may reduce the relative drift required for the onset of instability by a factor of approximately 30 , assuming the ion-acoustic speed of the medium remains constant. Therefore, the ion temperature anisotropy should be considered in future studies of ion-acoustic waves and instabilities in the high-latitude ionospheric F-region.
\end{abstract}

Keywords. Ionosphere (Plasma waves and instabilities)

\section{Introduction}

Gradients in perpendicular (horizontal) drift velocities, namely perpendicular shears, have been known to exist in the high latitude F-region for several decades. For instance, perpendicular shears as large as $20 \mathrm{~m} \mathrm{~s}^{-1} \mathrm{~m}^{-1}(\mathrm{~Hz})$ were mea- sured by a rocket experiment at the edges of nighttime auroral arcs (Kelley and Carlson, 1977), in conjunction with broadband low-frequency electrostatic waves. The largest perpendicular shears recorded in the literature were also uncovered from a rocket payload. In fact, broadband lowfrequency electrostatic waves associated with perpendicular shears of the order of $25 \mathrm{~Hz}$ were reported in the auroral $\mathrm{F}$ region (Earle et al., 1989).

Horizontal gradients in field-aligned (FA) flows, or parallel shears, are also known to exist in the high latitude Fregion. For example, shears in FA ion velocities were brought to notice with a rocket near and inside an auroral arc, at nighttime and concurrently with large perpendicular shears near the poleward boundary of an intense electron precipitation sector (Whalen et al., 1974). Satellite data from Dynamics Explorer (DE) 1 and 2 also showed evidence of parallel shears co-located with perpendicular shears (e.g. Basu et al., 1988; Loranc et al., 1991; Lu et al., 1992; Kivanc and Heelis, 1999, and the review by Koepke and Reynolds, 2007). The occurrence of simultaneous and co-located parallel and perpendicular shears suggests that the conditions behind one type of shears may be involved with the other one (Koepke and Reynolds, 2007). Interestingly, thermal ion upflows (TIU) appear to be located, at times, near auroral arc edges, in the same regions from which shears and large parallel current densities are observed (e.g. Tsunoda et al., 1989; St-Maurice and Hamza, 2009, and references therein).

In addition to field-aligned currents (FAC) and shears, ion temperature anisotropies can also be part of the picture. Often, the perpendicular temperature is seen to be larger than 
the parallel one $\left(T_{\perp \mathrm{i}}>T_{\| \mathrm{i}}\right)$. At low altitudes, one mechanism is the frictional heating of ions as they convect through the neutral gas, in presence of a DC electric field. A review of the theory of ion velocity distributions in the highlatitude F-region and the possible development of anisotropic ion temperatures in presence of perpendicular DC electric fields is presented by St-Maurice and Schunk (1979). The first experimental indication of non-Maxwellian distributions in the auroral F-region was provided by St-Maurice et al. (1976) using a retarding potential analyser onboard the Atmosphere Explorer C (AE-C) satellite. Later, anisotropic ion temperatures were observed in the high-latitude F-region, using the tri-static European Incoherent Scatter (EISCAT) UHF system, during strong convection electric field events (e.g. Perraut et al., 1984; Løvhaug and Flå, 1986). In particular, Løvhaug and Flå (1986) found ion anisotropy factors $\left(A_{\mathrm{i}}=T_{\perp \mathrm{i}} / T_{\| \mathrm{i}}\right)$ reaching 1.8 for the average situation and just above 2.5 in other cases. Likewise, EISCAT radar observations at a variety of aspect angles inferred similar ion temperature anisotropies. For instance, Winser et al. (1987) estimated an anisotropy factor $\left(A_{\mathrm{i}}\right)$ of at least 1.75 , assuming a line-of-sight Maxwellian distribution function. In the same way, Lockwood and Winser (1988) found anisotropies in excess of 2.

Another proposed mechanism for perpendicular ion heating is associated with plasma waves or instabilities. For example, simultaneous European Incoherent Scatter (EISCAT) Svalbard Radar (ESR) and EISCAT VHF observations showed that ion upflows can be, at times, linked with significant ion temperature anisotropy (e.g. Ogawa et al., 2000; Liu et al., 2004). In fact, Ogawa et al. (2000) suggested that waves contributed to heat ions horizontally up to an anisotropy ratio of $T_{\perp \mathrm{i}} / T_{\| \mathrm{i}} \sim 2$, for the event considered in their paper. Other authors proposed that velocity shear-driven instabilities could energise ions at lower altitudes and subsequently, drive ion upflows (Liu et al., 2004).

Numerical simulations also provided evidence of ion temperature anisotropies in the auroral ionosphere. For example, at low altitudes, a Monte-Carlo simulation involving $\mathrm{NO}^{+}$ions and a mixture of $\mathrm{O}$ and $\mathrm{N}_{2}$ neutrals resulted in an anisotropy factor up to approximately 1.3 depending on the electric field strength (Gaimard et al., 1998). Interestingly, the effects of shears in the convection flow on F-region ion velocity distributions, which were studied by St-Maurice et al. (1994), showed three-dimensional anisotropies. At higher altitudes in the topside, temperature anisotropies were also observed above the neutral exobase, which corresponds to the transition region from a weakly ionized plasma (ions collide with neutrals) to a fully ionized one (ions do not collide with neutrals), as a result of simulations from a single-component $\left(\mathrm{O}^{+}\right)$time-dependent gyro-kinetic model of the high-latitude F-region response to frictional heating, between $500 \mathrm{~km}$ and $2500 \mathrm{~km}$ (Loranc and St-Maurice, 1994). Namely, this ion upflow model simulates the response of the passage of a flux tube, under various con- ditions, through a spatially localised heating region for which the neutral exobase is a discontinuous boundary between fully collisional and collisionless plasmas. For one particular case, an anisotropy factor of $T_{\perp \mathrm{i}} / T_{\| \mathrm{i}} \sim 11$ was produced after $500 \mathrm{~s}$ and at an altitude of $1000 \mathrm{~km}$. Anisotropies with parallel enhancement were also observed. For the particular case under consideration, the ion parallel temperature increased sharply approximately $100 \mathrm{~s}$ after initial exobase heating up to transient values in excess of $2 \times 10^{4} \mathrm{~K}$ and then decreased abruptly at approximately $200 \mathrm{~s}$. Nonetheless, only temperature anisotropies with $T_{\perp \mathrm{i}}>T_{\| \mathrm{i}}$ are studied in this paper. Transient regimes with enhanced parallel temperatures could be addressed in future work. Thus, based on observational and numerical estimations, ion temperature anisotropies with $T_{\perp \mathrm{i}}>T_{\| \mathrm{i}}$ are plausible in the high latitude F-region.

Ion temperature anisotropies were also observed in laboratory experiments (Spangler et al., 2002, and references therein). Interestingly, laboratory experiments demonstrated that ion temperature anisotropy can significantly increase the growth rate of shear-driven ion-acoustic (IA) waves (Koepke, 2004; Teodorescu et al., 2003; Koepke et al., 2003). The increase in the growth rate with thermal anisotropy suggests that the plasma was unstable to smaller critical drifts.

Several competing plasma instabilities, triggered by FA drifts or horizontal shears in vertical velocity, have been invoked to explain high-latitude F-region irregularities observed by coherent or incoherent scatter radars. The role of transverse shears in FA ion drifts on electrostatic IA instability eigenmodes and threshold conditions was studied by Gavrishchaka et al. (1998) and Gavrishchaka et al. (1999). In particular, they suggested that velocity shears can significantly lower the threshold current of the current-driven electrostatic ion-acoustic (CDEIA) instability, even when $T_{\mathrm{i}} \sim T_{\mathrm{e}}$. Subsequently, St-Maurice et al. (2007) generalised Gavrishchaka et al. (1998)'s results by adding collisions with neutrals and finite Larmor radius corrections to the isotropic kinetic dispersion relation, which had not been considered by previous authors. In fact, St-Maurice et al. (2007) developed a generalised kinetic-based framework using a different derivation than Gavrishchaka et al. (1998), that is, using a local expansion of a drifting Maxwellian about a particular position in space. Later, Perron et al. (2009) systematically studied the threshold solutions of St-Maurice et al. (2007) kinetic dispersion relation for different ion to electron temperature ratios. Besides, the Gavrishchaka et al. (1998) and Gavrishchaka et al. (1999) work was extended by Spangler et al. (2002) who derived a dispersion relation for a Maxwellian plasma which includes temperature anisotropy and shears. They showed that ion temperature anisotropy alters the frequency modes of the sheared CDEIA instability by solving numerically the dispersion equation. They also demonstrated that ion thermal anisotropy increases the maximum growth rate, a conclusion which is consistent with laboratory results of Teodorescu et al. (2003). Spangler et al. (2002)'s findings involved numerical solutions of the 
dispersion relationship in terms of frequency and growth rate, but they did not consider threshold conditions.

The focus of the present work is to verify if ion temperature anisotropy in the topside F-region could lower the threshold conditions of ion shear CDEIA instabilities. These instabilitites could take place, for example, on the edges of auroral arcs, in presence of ion outflows and large parallel current densities. It appeared worthwhile to analyse marginally stable solutions because plasma destabilisation is already a challenging task by itself. Moreover, as far as Eregion radar observations are concerned, there is a tendency for the phase speed of the largest amplitude irregularities to saturate at the drift threshold, at least for structures less than $10 \mathrm{~m}$ in size (St-Maurice and Hamza, 2009). In order to first study the importance of shears and temperature anisotropy effects on threshold solutions, this communication explores auroral F-region situations where collisions with neutrals are negligible and for small finite Larmor radius corrections. The latter effects, in addition to temperature anisotropies and shears, will be presented in a subsequent paper. In Sect. 2, we introduce the kinetic dispersion relation. In Sect. 3, we present fluid-like modes in the limit $T_{\mathrm{e}} \gg T_{\perp \mathrm{i}}$ and compare with previous theories. Threshold solutions in the quasi-fluid limit are presented in Sect. 4. Finally, numerical results at higher ion to electron temperature ratios obtained from the kinetic dispersion relation are described in Sect. 5 and discussed in the context of F-region applications.

\section{Kinetic theory}

The electrostatic dispersion relationship for waves in a drifting collisional bi-Maxwellian plasma with sheared parallel ion flow and finite Lamor radius corrections, can be expressed as

$$
\frac{H_{\mathrm{i}}(k, \omega)}{1+U_{\mathrm{i}}(k, \omega)}+\frac{H_{\mathrm{e}}(k, \omega)}{1+U_{\mathrm{e}}(k, \omega)}+1=0
$$

where the ion susceptibility is given by

$$
\begin{aligned}
& H_{\mathrm{i}}=\frac{1}{k^{2} \lambda_{D i \perp}^{2}}\left[\frac{T_{\perp \mathrm{i}}}{T_{\| \mathrm{i}}}+\sum_{n} \Gamma_{n}\left(b_{\mathrm{i}}\right)\left(\frac{1}{\sqrt{2}\left|k_{\|}\right| v_{\| t i}}\right)\right. \\
& \left(n \Omega_{\mathrm{i}}\left(1-\frac{T_{\perp \mathrm{i}}}{T_{\| \mathrm{i}}}\right)+\omega \frac{T_{\perp \mathrm{i}}}{T_{\| \mathrm{i}}}\right) Z\left(\frac{\omega+i v_{\mathrm{i}}-n \Omega_{i}}{\sqrt{2}\left|k_{\|}\right| v_{\| t i}}\right)- \\
& \sum_{n} \Gamma_{n}^{*}\left(b_{i}\right) \frac{T_{\perp \mathrm{i}}}{T_{\| \mathrm{i}}} \frac{k_{\perp}}{k_{\|} \mid \Omega_{d i}^{\prime}}(1+ \\
& \left.\left.\frac{\omega+i v_{\mathrm{i}}-n \Omega_{\mathrm{i}}}{\sqrt{2}\left|k_{\|}\right| v_{\| t i}} Z\left(\frac{\omega+i v_{\mathrm{i}}-n \Omega_{\mathrm{i}}}{\sqrt{2}\left|k_{\|}\right| v_{\| t i}}\right)\right)\right] .
\end{aligned}
$$

For the electrons, since $\omega \ll \Omega_{\mathrm{e}}$, or equivalently $\rho_{L e}=$ $\sqrt{v_{t e} / \Omega_{\mathrm{e}}} \rightarrow 0$, we end up with

$$
\begin{aligned}
& H_{\mathrm{e}}=\frac{1}{k^{2} \lambda_{D i \perp}^{2}} \frac{T_{\perp \mathrm{i}}}{T_{\mathrm{e}}}[1+ \\
& \left.\left(\frac{\omega-k_{\|} V_{d e}+i v_{\mathrm{e}}}{\sqrt{2}\left|k_{\|}\right| v_{t e}}\right) Z\left(\frac{\omega-k_{\|} V_{d e}+i v_{\mathrm{e}}}{\sqrt{2}\left|k_{\|}\right| v_{t e}}\right)\right] .
\end{aligned}
$$

The expressions for $U_{\mathrm{i}}$ and $U_{\mathrm{e}}$, which arise from the inclusion of a BGK collision model in Boltzmann equation (StMaurice et al., 2007), are respectively

$$
U_{\mathrm{i}}=\frac{i v_{\mathrm{i}}}{\sqrt{2}\left|k_{\|}\right| v_{\| t i}}\left[\sum_{n} \Gamma_{n}\left(b_{\mathrm{i}}\right) Z\left(\frac{\omega+i v_{\mathrm{i}}-n \Omega_{\mathrm{i}}}{\sqrt{2}\left|k_{\|}\right| v_{\| t i}}\right)\right]
$$

and

$U_{\mathrm{e}}=\frac{i v_{\mathrm{e}}}{\sqrt{2}\left|k_{\|}\right| v_{t e}} Z\left(\frac{\omega-k_{z} V_{d e}+i v_{\mathrm{e}}}{\sqrt{2}\left|k_{\|}\right| v_{t e}}\right)$.

The previous dispersion relation was derived based on the following assumptions: electrostatic fluctuations and quasineutrality, isotropic electron temperature and the local approximation $\left(k_{x}=0\right)$ in the limit where the scale size of the shear is much larger than the wavelength. In addition, only ion velocity shears $\left(V_{d i}^{\prime}\right)$ are included in the previous dispersion equation since our aim is to describe instabilities associated with ion outflow processes. Furthermore, realistic electron velocity shears were seen to have a small effect on the zero shear solutions (Perron, 2004). The geometry of this model is presented in Fig. 1. In Eqs. (2) and (3), $\lambda_{D i \perp}$ is the ion perpendicular Debye length and $b_{\mathrm{i}}=$ $k_{\perp}^{2} v_{\perp t i}^{2} / \Omega_{\mathrm{i}}^{2}=k_{\perp}^{2} \rho_{L i}^{2}$ is the argument of the exponentially scaled modified Bessel function of the first kind $\left[\Gamma_{n}\left(b_{\mathrm{i}}\right)=\right.$ $\left.I_{n}\left(b_{\mathrm{i}}\right) e^{-b_{\mathrm{i}}}\right]$. The $\Gamma_{n}^{*}$ term is the new contribution produced by shears, given by $\Gamma_{n}^{*}\left(b_{\mathrm{i}}\right)=\left[\left(1-b_{\mathrm{i}}\right) I_{n}\left(b_{\mathrm{i}}\right)+b I_{n}^{\prime}\left(b_{\mathrm{i}}\right)\right] e^{-b_{\mathrm{i}}}$. The parallel and perpendicular thermal velocities for species $s$ are $v_{\| t s}^{2}=k_{B} T_{\| s} / m_{s}$ and $v_{\perp t s}^{2}=k_{B} T_{\perp s} / m_{s}$, respectively. $\Omega_{i}=q_{\mathrm{i}} B / m_{\mathrm{i}}$ is the ion gyrofrequency and $k^{2}=k_{\perp}^{2}+k_{\|}^{2}$ is the wave number. $V_{d e}$ is the electron parallel drift velocity with respect to the ion frame of reference (or electron-ion relative drift) and finally, $v_{\mathrm{i}, \mathrm{e}}$ is the ions (electrons) collision frequency with the neutrals. The full derivation of Eq. (1), which is a generalisation of St-Maurice et al. (2007) dispersion relation that includes ion temperature anisotropy, will be presented in a following paper. In this communication, we limit our study to the limiting case where $v_{\mathrm{i}, \mathrm{e}} \rightarrow 0$, or equivalently $U_{\mathrm{i}, \mathrm{e}} \rightarrow 0$, and for small Larmor radius corrections $\left(k_{\perp} \rho_{L i}=k_{\perp} v_{\perp t i}^{2} / \Omega_{\mathrm{i}}^{2} \ll 1\right)$, so that $\Gamma_{n}^{*} \approx \Gamma_{n}$. As a result, Eq. (1) is reduced to

$H_{\mathrm{i}}(k, \omega)+H_{\mathrm{e}}(k, \omega)+1=0$ 
where

$$
\begin{aligned}
& H_{\mathrm{i}}=\frac{1}{k^{2} \lambda_{D i \perp}^{2}}\left[\frac{T_{\perp \mathrm{i}}}{T_{\| i}}+\sum_{n} \Gamma_{n}\left(b_{\mathrm{i}}\right)\left(\frac{1}{\sqrt{2}\left|k_{\|}\right| v_{\| t i}}\right)\right. \\
& \left(n \Omega_{\mathrm{i}}\left(1-\frac{T_{\perp \mathrm{i}}}{T_{\| \mathrm{i}}}\right)+\omega \frac{T_{\perp \mathrm{i}}}{T_{\| \mathrm{i}}}\right) Z\left(\frac{\omega-n \Omega_{\mathrm{i}}}{\sqrt{2}\left|k_{\|}\right| v_{\| t i}}\right)- \\
& \left.\sum_{n} \Gamma_{n}\left(b_{\mathrm{i}}\right) \frac{T_{\perp \mathrm{i}}}{T_{\| \mathrm{i}}} \frac{k_{\perp} V_{d i}^{\prime}}{\left|k_{\|}\right| \Omega_{\mathrm{i}}}\left(1+\frac{\omega-n \Omega_{i}}{\sqrt{2}\left|k_{\|}\right| v_{\| t i}} Z\left(\frac{\omega-n \Omega_{\mathrm{i}}}{\sqrt{2}\left|k_{\|}\right| v_{\| t i}}\right)\right)\right]
\end{aligned}
$$

and

$$
H_{\mathrm{e}}=\frac{1}{k^{2} \lambda_{D i \perp}^{2}} \frac{T_{\perp \mathrm{i}}}{T_{\mathrm{e}}}\left[1+\left(\frac{\omega-k_{\|} V_{d e}}{\sqrt{2}\left|k_{\|}\right| v_{\| t e}}\right) Z\left(\frac{\omega-k_{\|} V_{d e}}{\sqrt{2}\left|k_{\|}\right| v_{\| t e}}\right)\right] .
$$

Note that the previous dispersion relation is equivalent to the one presented by Spangler et al. (2002). For the IA branch, $b_{\mathrm{i}} \ll 1$ and, therefore, the $n=0$ term dominates the series. In their analysis, Scime et al. (2003), Spangler et al. (2002) and Spangler (2001) reported that the $k^{2} \lambda_{D i \perp}$ term has a negligible effect on the dispersion relation solutions. We follow the same procedure and consider wavelengths much larger than the Debye radius in order to guarantee quasi-neutrality. We wish to stress, though, that this approximation is valid only if $\lambda \gg \lambda_{D i \perp}$, or equivalently, $\lambda_{\perp} \gg \lambda_{D i \perp}$ and $\lambda_{\|} \gg \lambda_{D i \perp}$ since $\lambda^{-2}=\lambda_{\perp}^{-2}+\lambda_{\|}^{-2}$. Otherwise, the anisotropy effect on threshold conditions would be rendered less important. Consequently, for the $n=0$ mode and for $k \lambda_{D i \perp} \rightarrow 0$, the ion temperature anisotropy effect is to increase the ion susceptibility $\left(H_{\mathrm{i}}\right)$ since each term is multiplied by $T_{\perp \mathrm{i}} / T_{\| \mathrm{i}}$. By contrast, the electron susceptibility $\left(H_{\mathrm{e}}\right)$ does not depend on ion anisotropy. It is worth noting that for $V_{d i}^{\prime} \rightarrow 0$, the linear dispersion relation for electrostatic waves in anisotropic magnetised hot plasmas is recovered (e.g. Stix, 1992, chap. 11).

\section{Fluid-like modes}

In their paper, Spangler et al. (2002), who added thermal anisotropy effects to Gavrishchaka et al. (1998)'s dispersion relation, derived a fluid-like mode after having performed the large ion and small electron argument expansions. They concluded that the fluid-like dispersion relation for ion-acoustic waves in an anisotropic plasma does not depend explicitly on ion temperature anisotropy. They essentially obtained an expression equivalent to the isotropic case except that the relevant electron temperature was the parallel one. However, by keeping higher order terms in the expansion, the threshold drift can have a thermal anisotropy dependency. For simplicity, we define $A_{\mathrm{i}}=T_{\perp \mathrm{i}} / T_{\| \mathrm{i}}$ and $\tau=T_{\perp \mathrm{i}} / T_{\mathrm{e}}$. Then, it can be shown that the critical relative drift (for zero growth rate condition), in the limit $\tau \ll 1$, can be expressed as

$\frac{V_{d e}}{C_{s}}=\frac{\omega_{\mathrm{R}}}{k_{\|} C_{s}}=\frac{\sqrt{1-\zeta_{i}}}{\sqrt{1+b_{s} A_{\mathrm{i}}}}$

where $\omega_{\mathrm{R}}$ is the real part of the frequency, $\zeta_{\mathrm{i}}=S_{\mathrm{i}} / \tan \theta$, $\tan \theta=\left|k_{\|}\right| / k_{\perp}, S_{\mathrm{i}}=V_{d i}^{\prime} / \Omega_{\mathrm{i}}$ and $b_{s}=\frac{k_{\perp}^{2} C_{s}^{2}}{\Omega_{\mathrm{i}}^{2}}=b_{\mathrm{i}} / \tau$. Note

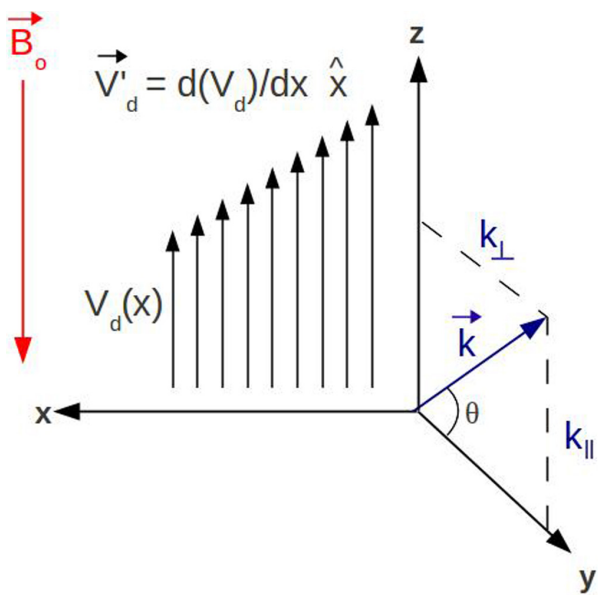

Fig. 1. Geometry of flow shear. The drift velocity is aligned with the magnetic field $\boldsymbol{B}_{\circ}$ and the gradient in the drift velocity is perpendicular to $\boldsymbol{B}_{\circ}$, in the X-direction. The wave vector $\boldsymbol{k}$ lies in the $\mathrm{y}-\mathrm{z}$ plane at an angle $\theta$ with respect to the direction perpendicular to $\boldsymbol{B}_{\circ}$. The perpendicular and parallel components of $\boldsymbol{k}$ are $k_{\perp}$ and $k_{\|}$.

that in the fluid-like limit $(\tau \ll 1)$, the ion-acoustic speed (Eq. 1 of Appendix A) reduces to $C_{s} \approx \sqrt{k_{B} T_{\mathrm{e}} / m_{\mathrm{i}}}$. The inclusion of ion anisotropy lowers the critical drift by a relatively small correction $\left(\sqrt{1+b_{s} A_{\mathrm{i}}}\right)$ in the denominator. When the ion anisotropy correction $\left(A_{\mathrm{i}}\right)$ is neglected, the fluid-like results of Gavrishchaka et al. (1998) and Spangler et al. (2002) are recovered. For shear-free and isotropic situations ( $\zeta_{\mathrm{i}}=0$ and $A_{\mathrm{i}} \rightarrow 1$ ), we find that $V_{d e} \sim C_{s}$ is required to destabilise the plasma, a threshold condition in agreement with the classical CDEIA instability (Kindel and Kennel, 1971).

\section{Numerical and analytical results in the quasi-fluid limit}

Equation (9) has been plotted in Fig. 2 (top panel) for different ion anisotropy factors and for $\omega_{\mathrm{R}} / k C_{s}=0.5$. We reduced the range of $S_{\mathrm{i}}$ and $V_{d e} / C_{s}$ in order to better observe the threshold variations with $A_{\mathrm{i}}$. We estimate that $\left|S_{\mathrm{i}}\right| \lesssim 0.1$ is plausible in the F-region. For example, with $1 \mathrm{~km} \mathrm{~s}^{-1}$ peak ion drifts along the magnetic field and $\mathrm{O}^{+}$ions, $\left|S_{\mathrm{i}}\right|=0.2$ would imply a horizontal shear scale on the order of $20 \mathrm{~m}$, which seems to be smaller than what might be possible by roughly one order of magnitude (Perron et al., 2009). That being stated, the threshold loci correspond to oblique waves with aspect angles in the approximate range of $15^{\circ} \lesssim \theta \lesssim$ $45^{\circ}$, with $\theta$ decreasing from the upper left corner to the lower right corner. The validity of Eq. (9) has been confirmed by solving the kinetic dispersion relation at low temperature ratios $(\tau)$ and within $\left|S_{\mathrm{i}}\right| \lesssim 1$. The exact numerical solutions were in excellent agreement with the analytical solutions for $A_{\mathrm{i}}=1,2,5$ and 10 , in the limit $\tau \lesssim 0.01$. For that reason, 

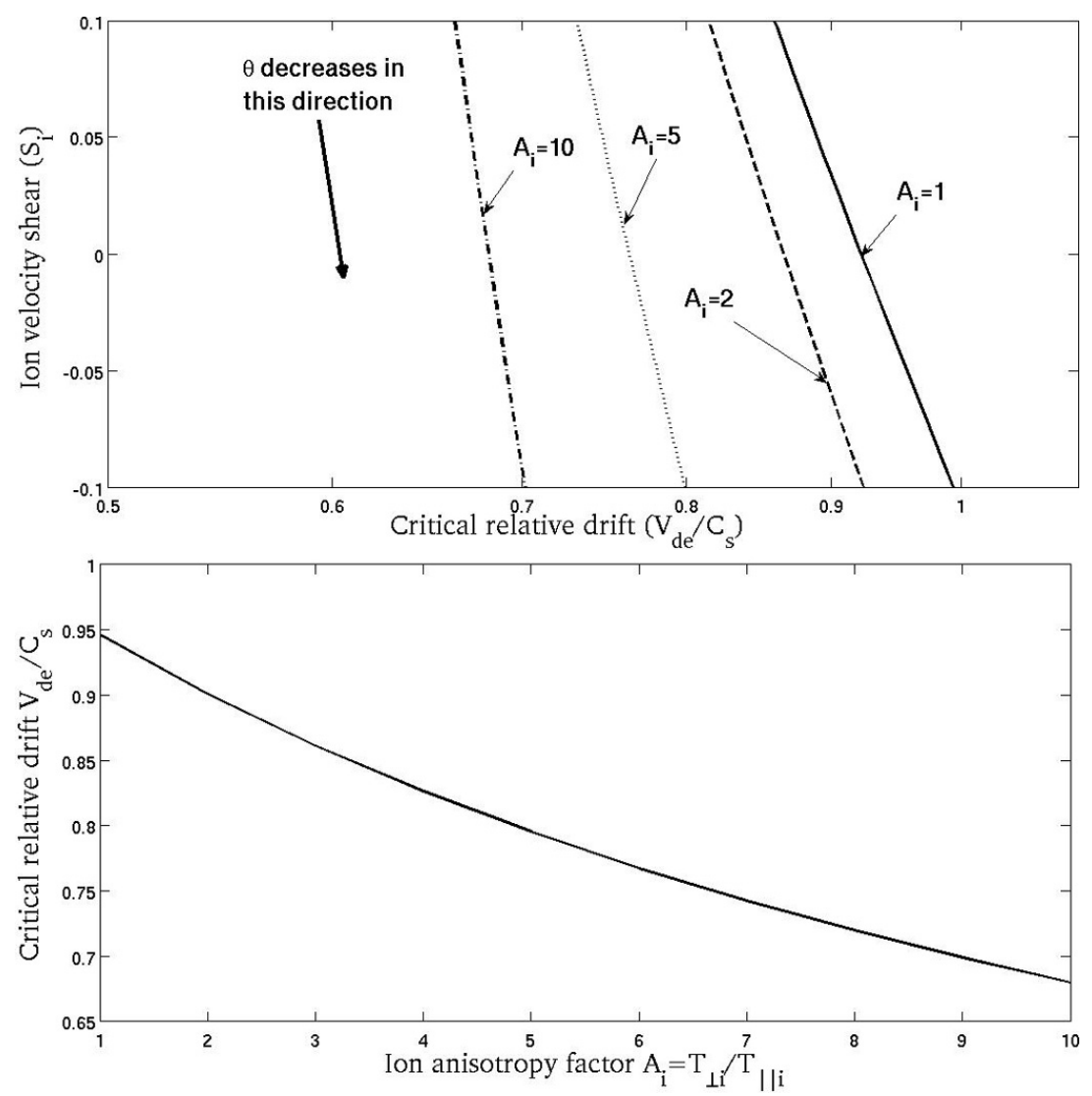

Fig. 2. Threshold conditions for the shear CDEIA instability in an anisotropic $\mathrm{O}^{+}$plasma using Eq. (9), $\omega_{\mathrm{R}} / k C_{S}=0.5$ and $\Omega_{\mathrm{i}} / k C_{S}=2 . S_{\mathrm{i}}$ as a function of $V_{d e} / C_{s}$ is presented in the top panel for $A_{\mathrm{i}}$ from 1 (solid), 2 (dashed), 5 (dotted) and 10 (dashed-dotted). Equation (9) is plotted in bottom panel for $\theta=47^{\circ}$ and $S_{\mathrm{i}}=0$.

kinetic solutions are not shown in Fig. 2. We notice a small decrease of critical drift with the ion anisotropy factor. This effect does not depend significantly on angle or ion shear, at least for the parameters considered herein, since the threshold curves remain almost parallel with respect to each other. We observe a critical drift reduction by a factor of approximately 0.2 to 0.3 depending on the wave vector angle, as $A_{\mathrm{i}}$ is increased from 1 to 10 and for a constant value of $C_{s}$. Put differently, this represents a 20 to $30 \%$ abatement compared with the isotropic threshold locus. The variation of drift threshold with ion anisotropy is illustrated in the lower panel of Fig. 2, in the case of zero shear $\left(S_{\mathrm{i}}=0\right)$ and for a fixed angle $\theta=47^{\circ}$. For this particular case, the drift threshold is lessened by approximately $28 \%$ as $A_{\mathrm{i}}$ varies from 1 to 10 . Indeed, we can conclude that ion temperature anisotropy acts to reduce the critical drift in the fluid-like limit, even in the presence of small or zero ion shear.

\section{Numerical kinetic results for larger temperature ra- tios $\tau$}

The kinetic solutions differ significantly from the quasi-fluid solutions when $\tau$ is larger than 0.1 , especially for large aspect angles. In this section, we investigate how $A_{\mathrm{i}}$ influences the kinetic threshold curves at an elevated ion over electron temperature ratio. A case study with $\tau=0.5$ is shown in Fig. 3 which was computed by finding the zeros of Eq. (1), with the help of Eqs. (2) and (3), using the same input frequencies as in Fig. 2 and for $\left|S_{\mathrm{i}}\right| \lesssim 1$. Note that shear values of $\left|S_{\mathrm{i}}\right| \sim 1$ should be considered with caution, since in this case, the ion shear magnitude $\left(V_{d i}^{\prime}\right)$ would be close to the ion gyrofrequency (St-Maurice et al., 2007). We observe that for a temperature ratio of $\tau=0.5$, ion temperature anisotropy affects significantly the threshold drift. For example, even with zero shear, an anisotropy factor of $A_{\mathrm{i}}=10$ acts to reduce the critical drift by a factor of approximately 100 to 200 for a constant $C_{s}$. When considering a more realistic ion anisotropy factor of $A_{\mathrm{i}}=2$ and a small negative shear $\left(S_{\mathrm{i}} \sim-0.1\right)$, the drift threshold $\left(V_{d e}\right)$ decreases by a factor of approximately 30 for a constant $C_{s}$. Therefore, ion temperature anisotropy is an important parameter to include 


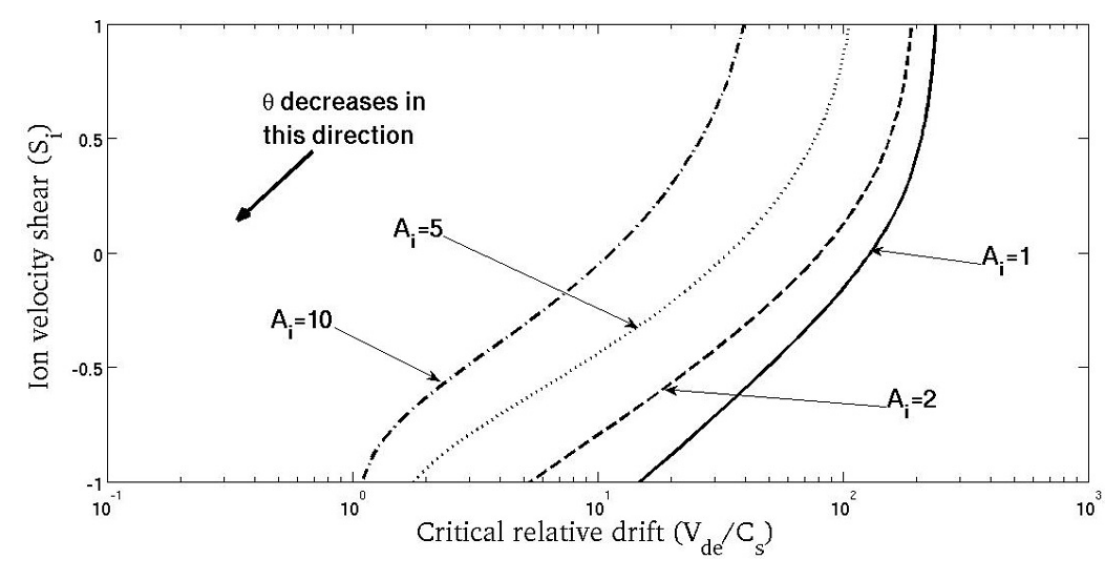

Fig. 3. Threshold conditions for the shear CDEIA instability in an anisotropic $\mathrm{O}^{+}$plasma using the kinetic dispersion relation (Eqs. 1, 2 and 3) for $A_{\mathrm{i}}$ from 1 (solid), 2 (dashed), 5 (dotted) and 10 (dashed-dotted), $\omega_{\mathrm{R}} / k C_{s}=0.5, \tau=0.5$ and $\Omega_{\mathrm{i}} / k C_{s}=2$.

in the kinetic dispersion relation since it can strongly reduce the critical current required to trigger ion-acoustic instabilities in a collisionless ionosphere.

\section{Conclusions}

We explored how the CDEIA marginal stability conditions (zero growth rate) in the long wavelength $\left(k \lambda_{D i \perp} \rightarrow 0\right)$ and small finite Larmor radius limits were affected by inclusion of ion velocity shears and ion temperature anisotropy. The approximate kinetic dispersion was derived assuming electrostatic modes, quasi-neutrality, isotropic electron temperature, zero electron velocity shears and the local approximation $\left(k_{x}=0\right)$ in the weak shear limit. All calculations were done for an $\mathrm{O}^{+}$collisionless plasma, which could resemble topside F-regions, at high enough altitudes where collisions could be safely neglected, but below approximately $1000 \mathrm{~km}$. We derived a new fluid-like expression which depends explicitly on ion temperature anisotropy. The addition of anisotropy factors actually lowers the critical drift in the quasi-fluid limit. Furthermore, when considering the full kinetic dispersion relation, it can significantly reduce the drift threshold. For example, an anisotropy factor of 2 lowered the electron drift $\left(V_{d e}\right)$ by a factor of approximately 30 assuming $C_{s}$ remains constant, for a small negative shear $\left(S_{\mathrm{i}} \sim-0.1\right)$ and $\tau=0.5$. Future work will involve adding collisions with neutrals and finite Larmor radius corrections to the anisotropic dispersion relation and studying its effects.

\section{Appendix A}

\section{Dimensionless quantities}

In this paper, we used normalisation parameters that differ from those of Spangler et al. (2002) since we were interested in comparing our results with St-Maurice et al. (2007)'s work. The critical drift numerical solutions were normalised with the total ion-acoustic speed, using the total ion temperature expression $T_{\mathrm{i}}=\left(T_{\| \mathrm{i}}+2 T_{\perp \mathrm{i}}\right) / 3$. Hence, the isothermal ion-acoustic speed can be formulated as a function of parallel and perpendicular thermal velocities

$$
\begin{aligned}
C_{s} & =v_{\| t i} \sqrt{A_{\mathrm{i}}\left(\frac{1}{\tau}+\frac{1}{3}\left(\frac{1}{A_{\mathrm{i}}}+2\right)\right)} \\
& =v_{t e} \sqrt{\frac{m_{\mathrm{e}}}{m_{\mathrm{i}}}} \sqrt{1+\frac{\tau}{3}\left(\frac{1}{A_{\mathrm{i}}}+2\right)} \\
& =v_{\perp t i} \sqrt{\frac{1}{\tau}+\frac{1}{3}\left(\frac{1}{A_{\mathrm{i}}}+2\right)}
\end{aligned}
$$

from which the following dimensionless quantities can be expressed as

$$
\begin{aligned}
& b_{\mathrm{i}}=\frac{k_{\perp}^{2} k_{B} T_{\perp \mathrm{i}}}{\Omega_{\mathrm{i}}^{2} m_{\mathrm{i}}}=\cos ^{2} \theta\left(\frac{k C_{s}}{\Omega_{\mathrm{i}}}\right)^{2}\left[\frac{1}{\left[\frac{1}{\tau}+\frac{1}{3}\left(\frac{1}{A_{\mathrm{i}}}+2\right)\right]}\right] \\
& \frac{\omega}{\sqrt{2}\left|k_{\|}\right| v_{\| t i}}=\left(\frac{\omega}{k C_{s}}\right)\left(\frac{1}{\sqrt{2} \sin \theta}\right) \sqrt{A_{\mathrm{i}}\left[\frac{1}{\tau}+\frac{1}{3}\left(\frac{1}{A_{\mathrm{i}}}+2\right)\right]} \\
& \frac{\omega}{\sqrt{2}\left|k_{\|}\right| v_{\| t e}}=\left(\frac{\omega}{k C_{s}}\right)\left(\frac{1}{\sqrt{2} \sin \theta}\right) \sqrt{\frac{m_{\mathrm{e}}}{m_{\mathrm{i}}}} \sqrt{1+\frac{\tau}{3}\left(\frac{1}{A_{\mathrm{i}}}+2\right)} \\
& \frac{V_{d e}}{\sqrt{2} v_{\| t e}}=\frac{V_{d e}}{C_{s}}\left(\frac{1}{\sqrt{2}}\right) \sqrt{\frac{m_{\mathrm{e}}}{m_{\mathrm{i}}}} \sqrt{1+\frac{\tau}{3}\left(\frac{1}{A_{\mathrm{i}}}+2\right) .}
\end{aligned}
$$

Acknowledgements. This work was supported by research grants from the National Sciences and Engineering Research Council of Canada.

Topical Editor K. Hosokawa thanks two anonymous referees for their help in evaluating this paper. 


\section{References}

Basu, S., Basu, S., MacKenzie, E., Fougere, P., Coley, W., Maynard, N., Winningham, J., Sugiura, M., Hanson, W., and Hoegy, W.: Simultaneous density and electric field fluctuation spectra associated with velocity shears in the auroral oval, J. Geophys. Res., 93, 115-136, 1988.

Earle, G., Kelley, M., and Ganguli, G.: Large velocity shears and associated electrostatic waves and turbulence in the auroral $\mathrm{F}$ region, J. Geophys. Res., 94, 15321-15333, 1989.

Gaimard, P., St-Maurice, J.-P., Lathuillere, C., and Hubert, D.: On the improvement of analytical calculations of collisional auroral ion velocity distributions using recent Monte Carlo results, J. Geophys. Res., 103, 4079-4095, 1998.

Gavrishchaka, V., Ganguli, S., and Ganguli, G.: Origin of lowfrequency oscillations in the ionosphere, Phys. Rev. Lett., 80, 728-731, 1998.

Gavrishchaka, V., Ganguli, S., and Ganguli, G.: Electrostatic oscillations due to filamentary structures in the magnetic-field-aligned flow: The ion-acoustic branch, J. Geophys. Res., 104, 1268312694, 1999.

Kelley, M. and Carlson, C.: Observations of intense velocity shear and associated electrostatic waves near an auroral arc, J. Geophys. Res., 82, 2343-2348, 1977.

Kindel, J. and Kennel, C.: Topside current instabilities, J. Geophys. Res., 76, 3055-3078, 1971.

Kivanc, O. and Heelis, R.: On relationships between horizontal velocity structure and thermal ion upwellings at high latitudes, Geophys. Res. Lett., 26, 1829-1832, 1999.

Koepke, M.: Sheared-flow-driven electrostatic waves in laboratory and space plasmas, Phys. Scr., 2004, 182-187, 2004.

Koepke, M. and Reynolds, E.: Simultaneous, co-located parallelflow shear and perpendicular-flow shear in low-temperature, ionospheric-plasma relevant laboratory plasma, Plasma Phys. Control. Fusion, 49, A145, doi:10.1088/0741-3335/49/5A/S12, 2007.

Koepke, M., Teodorescu, C., and Reynolds, E.: Space relevant laboratory studies of ion-acoustic and ion-cyclotron waves driven by parallel-velocity shear, Plasma Phys. Control. Fusion, 45, 869889, 2003.

Liu, H. and Lu, G.: Velocity shear-related ion upflow in the low-altitude ionosphere, Ann. Geophys., 22, 1149-1153, doi:10.5194/angeo-22-1149-2004, 2004.

Lockwood, M. and Winser, K.: On the determination of ion temperature in the auroral F-region ionosphere, Planet. Space Sci., 36, 1295-1304, 1988.

Loranc, M. and St-Maurice, J.-P.: A time-dependent gyro-kinetic model of thermal ion upflows in the high-latitude $\mathrm{F}$ region, J. Geophys. Res., 99, 17429-17451, 1994.

Loranc, M., Hanson, W., Heelis, R., and St-Maurice, J.-P.: A morphological study of vertical ionospheric flows in the high-latitude F region, J. Geophys. Res., 96, 3627-3646, 1991.

Løvhaug, U. and Flå, T.: Ion temperature anisotropy in the auroral F-region as measured with EISCAT, J. Atmos. Terr. Phys., 48, 959-971, 1986.

Lu, G., Reiff, P., Moore, T., and Heelis, R.: Upflowing ionospheric ions in the auroral region, J. Geophys. Res., 97, 16855-16863, 1992.
Ogawa, Y., Fujii, R., Buchert, S., Nozawa, S., Watanabe, S., and Van Eyken, A.: Simultaneous EISCAT Svalbard and VHF radar observations of ion upflows at different aspect angles, Geophys. Res. Lett., 27, 81-84, 2000.

Perraut, S., Brekke, A., Baron, M., and Hubert, D.: EISCAT measurements of ion temperatures which indicate non-isotropic ion velocity distributions, J. Atmos. Terr. Phys., 46, 531-543, 1984.

Perron, P. J.: Shear and current driven electrostatic ion-acoustic instability in a collisional ionosphere: Numerical analysis of the fluid and kinetic dispersion relations, Master's thesis, Royal Military College of Canada, Kingston, Ontario, 2004.

Perron, P. J. G., Noël, J.-M. A., and St.-Maurice, J.-P.: Velocity shear and current driven instability in a collisional F-region, Ann. Geophys., 27, 381-394, doi:10.5194/angeo-27-381-2009, 2009.

Scime, E., Murphy, R., Ganguli, G., and Edlund, E.: Electrostatic ion-cyclotron waves in a currentless, anisotropic plasma with inhomogeneous flow, Phys. Plasmas, 10, 4609-4612, 2003.

Spangler, R. S.: A kinetic treatment of a perpendicular gradient in field-aligned flow in a thermally anisotropic plasma, Master's thesis, West Virginia University, 2001.

Spangler, R., Scime, E., and Ganguli, G.: Parallel inhomogeneous flows in a thermally anisotropic plasma: The electrostatic ionacoustic branch, Phys. Plasmas, 9, 2526-2533, 2002.

St-Maurice, J.-P. and Hamza, A.: Small scale irregularities at high latitudes, Characterising the ionopshere, edited by: Wyman, G.; Technical Report RTO-TR-IST-051, http://www.rto.nato.int/ abstracts.aspx, 2009.

St-Maurice, J.-P. and Schunk, R.: Ion velocity distributions in the high-latitude ionosphere, Rev. Geophys. Space Phys., 17, 99133, 1979.

St-Maurice, J., Hanson, W., and Walker, J.: Retarding potential analyzer measurement of the effect of ion-neutral collisions on the ion velocity distribution in the auroral ionosphere, J. Geophys. Res., 81, 5438-5446, 1976.

St-Maurice, J., Winkler, E., and Hamza, A.: Ionospheric ion velocity distributions and associated transport properties in the presence of auroral electric field gradients, J. Geophys. Res., 99, 19527-19549, 1994.

St-Maurice, J., Noël, J.-M., and Perron, P.: An assessment of how a combination of shears, field-aligned currents and collisions affect F-region ionospheric instabilities, J. Plasma Physics, 73, 69-88, 2007.

Stix, T.: Waves in plasmas, American Institute of Physics, 1992.

Teodorescu, C., Koepke, M., and Reynolds, E.: On the role of ion temperature anisotropy in the growth and propagation of shearmodified ion-acoustic waves, J. Geophys. Res., 108, 1043-1053, 2003.

Tsunoda, R., Livingston, R., Vickrey, J., Heelis, R., Hanson, W., Rich, F., and Bythrow, P.: Dayside observations of thermal-ion upwellings at $800-\mathrm{km}$ altitude: an ionospheric signature of the cleft ion fountain, J. Geophys. Res., 94, 15277-15290, 1989.

Whalen, B., Green, D., and McDiarmid, I.: Observations of ionospheric ion flow and related convective electric fields in and near an auroral arc, J. Geophys. Res., 79, 2835-2842, 1974.

Winser, K., Lockwood, M., and Jones, G.: Non-thermal plasma observations using EISCAT: Aspect angle dependence, Geophys. Res. Lett., 14, 957-960, 1987. 